\title{
Erratum: Tritium $\beta$ decay in chiral effective field theory [Phys. Rev. C 94, 024003 (2016)]
}

\author{
A. Baroni, L. Girlanda, A. Kievsky, L. E. Marcucci, R. Schiavilla, and M. Viviani \\ (Received 30 December 2016; published 12 May 2017)
}

DOI: 10.1103/PhysRevC.95.059902

An error in the loop function $W_{3}(k)$ reported in Eq. (D12) of Ref. [1] has been corrected in the Erratum [2]. The corrected expression for $W_{3}(k)$ in Eq. (20) of the original paper as obtained in the Erratum [2] reads

$$
W_{3}(k)=-\frac{1}{2} \int_{0}^{1} d z \frac{1}{M(k, z)} .
$$

As a result of this error the following minor changes to the tritium $\beta$ decay results reported in the original paper are obtained:

(1) The values reported in the row labeled N4LO (MPE) [(next-to-next-to-next-to-next-to-leading order (multipion exchange)] of Table I of the original paper (the only ones affected by the error) change as follows:

(2) The values in the two columns under the heading N4LO in Table II of the original paper (the only ones affected by the error) change as follows:

(3) The values in the two columns under the heading N4LO in Table III of the original paper (the only ones affected by the error) change as follows:

(4) Figures 4 and 5 change as follows (the vertical lines corresponding to the N4LO calculations are slightly shifted relative to the original figures):

(5) Figure 7 changes as follows:

These changes do not affect any of the conclusions of the original paper. In particular, the results for the $n d$ doublet scattering lengths given in the bottom left column of p. 8 of the original paper are within 0.001 fm of those listed there when the correct values of $\left(c_{D}, c_{E}\right)$ from item (3) above are used.

TABLE I.

\begin{tabular}{lcc}
\hline \hline$\Lambda$ & $500 \mathrm{MeV}$ & $600 \mathrm{MeV}$ \\
\hline N4LO (MPE) & $-0.416(-0.552) \times 10^{-1}$ & $-0.513(-0.730) \times 10^{-1}$ \\
\hline \hline
\end{tabular}

TABLE II.

\begin{tabular}{lrr}
\hline \hline & \multicolumn{2}{c}{ N4LO } \\
\cline { 2 - 3 }$\Lambda$ & \multicolumn{1}{c}{500} & 600 \\
\hline$\hat{z}_{0}$ & -1.586 & -0.962 \\
$\hat{d}_{R}$ & 0.959 & 1.584 \\
$c_{D}$ & -2.150 & -1.303 \\
$\hat{z}_{0}^{\star}$ & -0.395 & 0.322 \\
$\hat{d}_{R}^{\star}$ & 0.682 & 1.400 \\
$c_{D}^{\star}$ & -0.535 & 0.437 \\
\hline \hline
\end{tabular}

TABLE III.

\begin{tabular}{lcc}
\hline \hline & \multicolumn{2}{c}{ N4LO } \\
\cline { 2 - 3 }$\Lambda$ & 500 & 600 \\
\hline$c_{D}$ & -1.806 & -1.982 \\
$c_{E}$ & -0.542 & -1.542 \\
\hline \hline
\end{tabular}

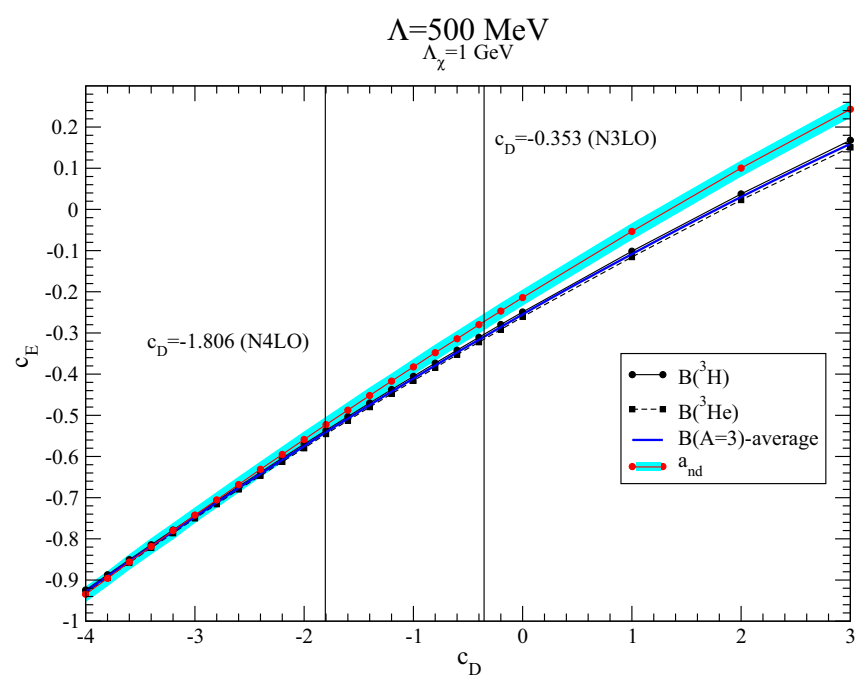

FIG. 4. The vertical line that indicates the $c_{D}$ values obtained by fitting the $\mathrm{GT}_{\text {expt. }}$ (where GT represents Gamow-Teller) retaining N4LO has been corrected. 


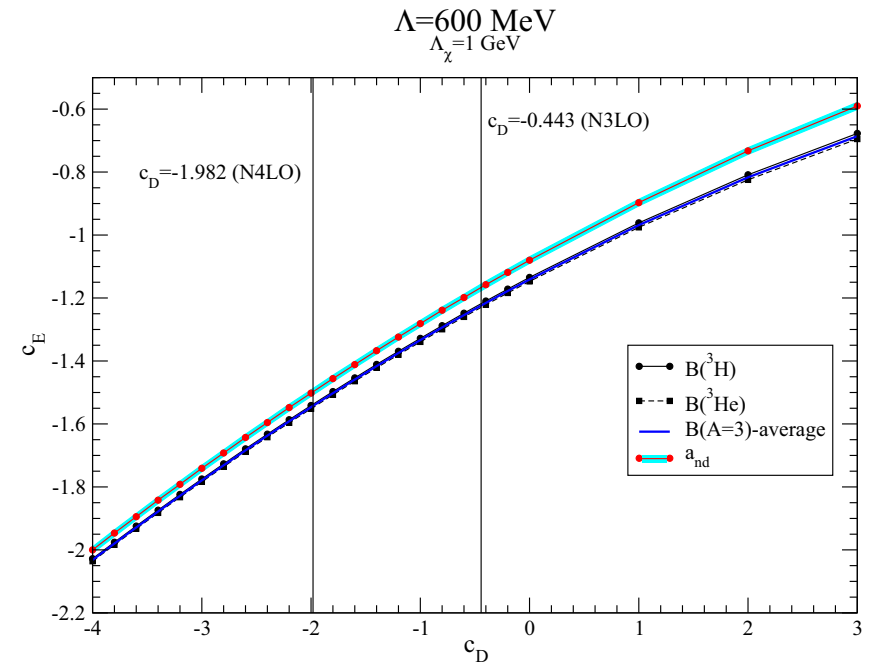

FIG. 5. The same as Fig. 4 but for $\Lambda=600 \mathrm{MeV}$.

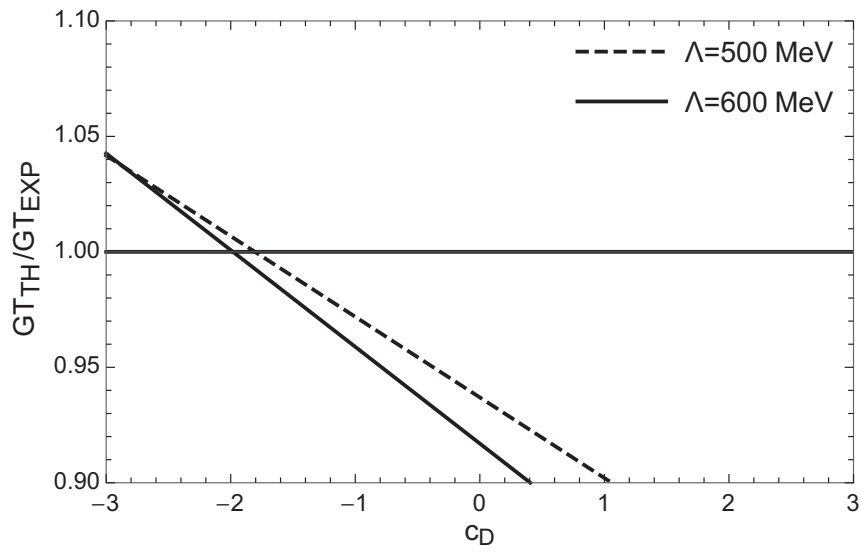

FIG. 7. The ratio $\mathrm{GT}_{\text {th }} / \mathrm{GT}_{\text {expt. }}$ as a function of the low-energy constant $c_{D}$ obtained retaining corrections up to N4LO in the axial current has been corrected for both values of the cutoff $\Lambda$.

[1] A. Baroni, L. Girlanda, S. Pastore, R. Schiavilla, and M. Viviani, Phys. Rev. C 93, 015501 (2016); 93, 049902(E) (2016).

[2] A. Baroni, L. Girlanda, S. Pastore, R. Schiavilla, and M. Viviani, preceding Erratum, Phys. Rev. C 95, 059901 (2017). 